\title{
New Generalized Hyperbolic Functions to Find New Exact Solutions of the Nonlinear Partial Differential Equations
}

\author{
Yusuf Pandir and Halime Ulusoy \\ Department of Mathematics, Faculty of Science, Bozok University, 66100 Yozgat, Turkey \\ Correspondence should be addressed to Yusuf Pandir; yusufpandir@gmail.com
}

Received 2 October 2012; Accepted 30 October 2012

Academic Editor: Anjan Biswas

Copyright (C) 2013 Y. Pandir and H. Ulusoy. This is an open access article distributed under the Creative Commons Attribution License, which permits unrestricted use, distribution, and reproduction in any medium, provided the original work is properly cited.

We firstly give some new functions called generalized hyperbolic functions. By the using of the generalized hyperbolic functions, new kinds of transformations are defined to discover the exact approximate solutions of nonlinear partial differential equations. Based on the generalized hyperbolic function transformation of the generalized KdV equation and the coupled equal width wave equations (CEWE), we find new exact solutions of two equations and analyze the properties of them by taking different parameter values of the generalized hyperbolic functions. We think that these solutions are very important to explain some physical phenomena.

\section{Introduction}

To find exact solutions to nonlinear evolution equations, some new techniques have been introduced in recent years such as the Hirota method, the extended Jacobi elliptic function expansion method, the exponential function method, $\left(G^{\prime} / G\right)$-expansion method, the simplest equation method, the trial equation method, Kudryashov's method, and solitary ansatz method [1-19]. Also some authors define new functions which are named generalized hyperbolic functions for constructing new solutions [20-22]. There are a lot of nonlinear evolution equations that are integrated using the various mathematical methods. Soliton solutions, compactons, singular solitons, and other solutions have been found by using these approaches. These types of solutions are very important and appear in various areas of applied mathematics.

In Section 2, we give the definition and properties of generalized hyperbolic functions. In Section 3, as applications, we obtain exact solutions of the generalized KdV equation and the coupled equal width wave equations.

\section{The Definition and Properties of the Symmetrical Hyperbolic Fibonacci and Lucas Functions}

In this section, we will define new functions which are named the symmetrical hyperbolic Fibonacci and Lucas functions for constructing new exact solutions of NPDEs and then study the properties of these functions.

Definition 1. Suppose that $\xi$ is an independent variable; $p$, $q$, and $k$ are all constants. The generalized hyperbolic sine function is

$$
\sinh _{a}(\xi)=\frac{p a^{k \xi}-q a^{-k \xi}}{2},
$$

generalized hyperbolic cosine function is

$$
\cosh _{a}(\xi)=\frac{p a^{k \xi}+q a^{-k \xi}}{2},
$$

generalized hyperbolic tangent function is

$$
\tanh _{a}(\xi)=\frac{p a^{k \xi}-q a^{-k \xi}}{p a^{k \xi}+q a^{-k \xi}},
$$


generalized hyperbolic cotangent function is

$$
\operatorname{coth}_{a}(\xi)=\frac{p a^{k \xi}+q a^{-k \xi}}{p a^{k \xi}-q a^{-k \xi}},
$$

generalized hyperbolic secant function is

$$
\operatorname{sech}_{a}(\xi)=\frac{2}{p a^{k \xi}+q a^{-k \xi}},
$$

generalized hyperbolic cosecant function is

$$
\operatorname{cosech}_{a}(\xi)=\frac{2}{p a^{k \xi}-q a^{-k \xi}},
$$

and the above six kinds of functions are said to be generalized new hyperbolic functions. Thus we can prove the following theory of generalized hyperbolic functions on the basis of Definition 1.

Theorem 2. The generalized hyperbolic functions satisfy the following relations:

$$
\begin{gathered}
\cosh _{a}^{2}(\xi)-\sinh _{a}^{2}(\xi)=p q, \\
1-\tanh _{a}^{2}(\xi)=p q \operatorname{sech}_{a}^{2}(\xi), \\
1-\operatorname{coth}_{a}^{2}(\xi)=-p q \operatorname{cosech}_{a}^{2}(\xi), \\
\operatorname{sech}_{a}(\xi)=\frac{1}{\cosh _{a}(\xi)}, \\
\operatorname{cosech}_{a}(\xi)=\frac{1}{\sinh _{a}(\xi)}, \\
\tanh _{a}(\xi)=\frac{\sinh _{a}(\xi)}{\cosh _{a}(\xi)}, \\
\operatorname{coth}_{a}(\xi)=\frac{\cosh _{a}(\xi)}{\sinh _{a}(\xi)} .
\end{gathered}
$$

The following just part of them are proved here for simplification.

Theorem 3. The derivative formulae of generalized hyperbolic functions are as follows:

$$
\begin{gathered}
\frac{d\left(\sinh _{a}(\xi)\right)}{d \xi}=k \ln a \cosh _{a}(\xi), \\
\frac{d\left(\cosh _{a}(\xi)\right)}{d \xi}=k \ln a \sinh _{a}(\xi), \\
\frac{d\left(\tanh _{a}(\xi)\right)}{d \xi}=k p q \ln a \operatorname{sech}_{a}^{2}(\xi), \\
\frac{d\left(\operatorname{coth}_{a}(\xi)\right)}{d \xi}=-k p q \ln a \operatorname{cosech}_{a}^{2}(\xi), \\
\frac{d\left(\operatorname{sech}_{a}(\xi)\right)}{d \xi}=-k \ln a \operatorname{sech}_{a}(\xi) \tanh _{a}(\xi), \\
d \xi
\end{gathered}
$$

Proof of (8). According to (7) and (8), we can get

$$
\begin{aligned}
\frac{d\left(\tanh _{a}(\xi)\right)}{d \xi} & =\left(\frac{\sinh _{a}(\xi)}{\cosh _{a}(\xi)}\right)^{\prime} \\
& =\frac{\left(\sinh _{a}(\xi)\right)^{\prime} \cosh _{a}(\xi)-\left(\cosh _{a}(\xi)\right)^{\prime} \sinh _{a}(\xi)}{\cosh _{a}^{2}(\xi)} \\
& =\frac{k \ln a \cosh _{a}^{2}(\xi)-k \ln a \sinh _{a}^{2}(\xi)}{\cosh _{a}^{2}(\xi)} \\
& =k p q \ln a \operatorname{sech}_{a}^{2}(\xi)
\end{aligned}
$$

Similarly, we can prove other differential coefficient formulae in Theorem 3.

Remark 4. We see that when $p=1, q=1, k=1$, and $a=e$ in (1)-(6), new generalized hyperbolic functions $\sinh _{a}(\xi)$, $\cosh _{a}(\xi), \tanh _{a}(\xi), \operatorname{coth}_{a}(\xi), \operatorname{sech}_{a}(\xi)$, and $\operatorname{cosech}_{a}(\xi)$ degenerate as hyperbolic functions $\sinh (\xi), \cosh (\xi), \tanh (\xi)$, $\operatorname{coth}(\xi), \operatorname{sech}(\xi)$, and $\operatorname{cosech}(\xi)$, respectively. In addition, when $p=0$ or $q=0$ in (1)-(6), $\sinh _{a}(\xi), \cosh _{a}(\xi), \tanh _{a}(\xi)$, $\operatorname{coth}_{a}(\xi), \operatorname{sech}_{a}(\xi)$, and $\operatorname{cosech}_{a}(\xi)$ degenerate as exponential function $(1 / 2) p a^{k(\xi)}, \pm(1 / 2) q a^{-k(\xi)}, 2 p a^{-k(\xi)}, \pm 2 q a^{k(\xi)}$, and \pm 1 , respectively.

\section{Applications}

Example 5. Application to the generalized form of $\mathrm{KdV}$ equation is presented.

The generalized KdV equation that will be studied in this paper are given by [23]

$$
\left(u^{l}\right)_{t}+\alpha u^{n} u_{x}+\beta\left(u^{n} u_{x x}\right)_{x}+\gamma u\left(u^{n}\right)_{x x x}=0
$$

where the first term represents the generalized evolution. The special case with $l=1$ is the regular evolution term. The coefficients of $\alpha$ are the nonlinear terms while the coefficients of $\beta$ and $\gamma$ are the nonlinear dispersion terms. This equation with $l=1$ has been already studied by [18], where, in addition to soliton solution, compactons and periodic solutions were also obtained. The hypothesis for solving this equation is

$$
u(x, t)=\frac{A}{\cosh _{a}^{s}(\eta)},
$$

where

$$
\eta=B(x-v t)
$$

Here, in (11) and (12), $A$ represents the amplitude of the soliton while $B$ is the inverse width of the soliton and $v$ is the velocity of the soliton. The exponent $s$ is unknown at this point and will be evaluated during the course of 
the derivation of the solutions to (10). From (11), it is possible to obtain

$$
\begin{gathered}
\left(u^{l}\right)_{t}=\frac{k l s v A^{l} B \ln a \tanh _{a}(\eta)}{\cosh _{a}^{s l}(\eta)}, \\
u^{n} u_{x}=\frac{-k s A^{n+1} B \ln a \tanh _{a}(\eta)}{\cosh _{a}^{s(n+1)}(\eta)}, \\
\left(u^{n} u_{x x}\right)_{x} \\
=\frac{k^{3} s A^{n+1} B^{3} p q(\ln a)^{3}(s+1)(s n+s+2) \tanh _{a}(\eta)}{\cosh _{a}^{s(n+1)+2}(\eta)} \\
u\left(u^{n}\right)_{x x x} \frac{k^{3} s^{3}(n+1) A^{n+1} B^{3}(\ln a)^{3} \tanh (\eta)}{\cosh _{a}^{s(n+1)}(\eta)}, \\
=\frac{-k^{3} s^{3} n^{3} A^{n+1} B^{3}(\ln a)^{3} \tanh _{a}(\eta)}{\cosh _{a}^{s(n+1)}(\eta)} \\
+\frac{k^{3} s n p q A^{n+1} B^{3}(\ln a)^{3}(s n+1)(s n+2) \tanh _{a}(\eta)}{\cosh _{a}^{s(n+1)+2}(\eta)} .
\end{gathered}
$$

These results will now be substituted in (10) to obtain the 1soliton solution of the generalized $\mathrm{KdV}$ equation. Equation (10) by virtue of (13) reduces to

$$
\begin{aligned}
& \frac{k l s v A^{l} B \ln a \tanh _{a}(\eta)}{\cosh _{a}^{s l}(\eta)}-\frac{\alpha k s A^{n+1} B \ln a \tanh (\eta)}{\cosh _{a}^{s(n+1)}(\eta)} \\
& +\frac{\beta k^{3} s p q A^{n+1} B^{3}(\ln a)^{3}(s+1)(s n+s+2) \tanh _{a}(\eta)}{\cosh _{a}^{s(n+1)+2}(\eta)} \\
& -\frac{\beta k^{3} s^{3}(n+1) A^{n+1} B^{3}(\ln a)^{3} \tanh (\eta)}{\cosh _{a}^{s(n+1)}(\eta)} \\
& +\frac{\gamma k^{3} \operatorname{sn}^{2} p A^{n+1} B^{3}(\ln a)^{3}(s n+1)(s n+2) \tanh _{a}(\eta)}{\cosh _{a}^{s(n+1)+2}(\eta)} \\
& -\frac{\gamma k^{3} s^{3} n^{3} A^{n+1} B^{3}(\ln a)^{3} \tanh _{a}(\eta)}{\cosh _{a}^{s(n+1)}(\eta)}=0 .
\end{aligned}
$$

From (14), equating the exponents $s l$ and $s n+s+2$ gives

$$
s l=s n+s+2,
$$

that leads to

$$
s=\frac{2}{l-n-1} .
$$

Now from (14), the two linearly independent functions are $1 / \cosh ^{n s+s+j}$ for $j=0,2$. Thus setting their coefficients to zero gives

$$
\begin{gathered}
v=\frac{\alpha p q(\beta l(l+1-n)+n \gamma(l-1)(l+n-1))}{2 l A^{l-n-1}\left(\beta(n+1)+n^{3} \gamma\right)}, \\
B=\frac{\sqrt{-\alpha}(n+1-l)}{2 k \ln a \sqrt{\beta(n+1)+n^{3} \gamma}} .
\end{gathered}
$$

Thus the 1-soliton solution of the generalized KdV equation with generalized evolution is given by

$$
u(x, t)=\frac{A}{\cosh _{a}^{2 /(l-n-1)}[B(x-v t)]} .
$$

This shows that the restriction on the nonlinear exponents of (10) must be

$$
l>n+1
$$

Remark 6. If we take the corresponding values for some parameters, the solution (19) can be reduced to the solution (17) mentioned in [23].

Example 7. Application to the coupled equal width wave equations is presented.

We consider the coupled equal width wave equations [24]

$$
\begin{aligned}
& u_{t}+u u_{x}-u_{x x t}+r r_{x}=0, \\
& r_{t}+r r_{x}-r_{x x t}=0 .
\end{aligned}
$$

The hypothesis for solving these equations is

$$
\begin{aligned}
& r(x, t)=\frac{A_{1}}{\cosh _{a}^{s_{1}}(\eta)}, \\
& u(x, t)=\frac{A_{2}}{\cosh _{a}^{s_{2}}(\eta)},
\end{aligned}
$$

where

$$
\eta=B(x-v t)
$$

Here in (22)-(23) $A_{1}$ and $A_{2}$ are the amplitudes of the $r$ soliton and $u$-soliton, respectively, while $v_{1}$ and $v_{2}$ are the velocity of the soliton and $B_{1}$ and $B_{2}$ are the inverse widths of the solitons. The exponents $s_{1}$ and $s_{2}$ are unknown at this 
point and their values will fall out in the process of deriving the solution of this equation. From (22), it is possible to obtain

$$
\begin{aligned}
& u_{t}= \frac{k s_{2} v A_{2} B \ln a \tanh _{a}(\eta)}{\cosh _{a}^{s_{2}}(\eta)}, \\
& r_{t}= \frac{k s_{1} v A_{1} B \ln a \tanh _{a}(\eta)}{\cosh _{a}^{s_{1}}(\eta)}, \\
& u_{x}=\frac{-k s_{2} A_{2} B \ln a \tanh _{a}(\eta)}{\cosh _{a}^{s_{2}}(\eta)} \\
& r_{x}=\frac{-k s_{1} A_{1} B \ln a \tanh (\eta)}{\cosh _{a}^{s_{1}}(\eta)}, \\
& u_{x x t}=\frac{k^{3} s_{2}^{3} v A_{2} B^{3}(\ln a)^{3} \tanh _{a}(\eta)}{\cosh _{a}^{s_{2}}(\eta)} \\
& r_{x x t}=\frac{k^{3} s_{2} p q\left(s_{2}+1\right) v A_{2} B^{3}(\ln a)^{3}\left(s_{2}+2\right) \tanh _{a}(\eta)}{\cosh _{a}^{s_{2}+2}(\eta)} \\
& \cosh _{a}^{3} A_{1}^{3}(\ln a)^{3} \tanh _{a}(\eta) \\
& \cosh _{a}^{s_{1}}(\eta)
\end{aligned}
$$

Substituting these into (21) yields to

$$
\begin{aligned}
& \frac{k s_{2} v A_{2} B \ln a \tanh _{a}(\eta)}{\cosh _{a}^{s_{2}}(\eta)}-\frac{k s_{2} A_{2}^{2} B \ln a \tanh _{a}(\eta)}{\cosh _{a}^{2 s_{2}}(\eta)} \\
& -\frac{k^{3} s_{2}^{3} v A_{2} B^{3}(\ln a)^{3} \tanh _{a}(\eta)}{\cosh _{a}^{s_{2}}(\eta)} \\
& +\frac{k^{3} s_{2}\left(s_{2}+1\right)\left(s_{2}+2\right) p q v A_{2} B^{3}(\ln a)^{3} \tanh _{a}(\eta)}{\cosh _{a}^{s_{2}+2}(\eta)} \\
& -\frac{k s_{1} A_{1}^{2} B \ln a \tanh _{a}(\eta)}{\cosh _{a}^{2 s_{1}}(\eta)}=0 \\
& \frac{k s_{1} v A_{1} B \ln a \tanh _{a}(\eta)}{\cosh _{a}^{s_{1}}(\eta)}-\frac{k s_{1} A_{1}^{2} B \ln a \tanh (\eta)}{\cosh _{a}^{2 s_{1}}(\eta)} \\
& -\frac{k^{3} s_{1}^{3} v A_{1} B^{3}(\ln a)^{3} \tanh _{a}(\eta)}{\cosh _{a}^{s_{1}}(\eta)} \\
& +\frac{k^{3} s_{1}\left(s_{1}+1\right)\left(s_{1}+2\right) v p q A_{1} B^{3}(\ln a)^{3} \tanh _{a}(\eta)}{\cosh _{a}^{s_{1}+2}(\eta)}=0
\end{aligned}
$$

Now from (28) and (29), equating the exponents $2 s_{1}$ and $s_{1}+2$ and also $2 s_{2}$ and $s_{2}+2$ gives, respectively,

$$
2 s_{1}=s_{1}+2, \quad 2 s_{2}=s_{2}+2,
$$

that leads to

$$
s_{1}=s_{2}=2 \text {. }
$$

So from (28) and (29), the four linearly independent functions are $1 / \cosh ^{s_{1}+j}$ and $1 / \cosh ^{s_{2}+j}$ for $j=0,2$. Therefore, setting their respective coefficients to zero we obtain $A_{2}, B$, and $v$ as follow.

Case 1. One has

$$
A_{2}=\frac{A_{1}}{2}(1 \pm i \sqrt{3}), \quad B=-\frac{1}{2 k \ln a}, \quad v=\frac{A_{1}}{3 p q},
$$

where $A_{1}, k, p$, and $q$ are arbitrary constants. Substituting (32) into (22), we obtain new exact solution to (21),

$$
r(x, t)=\frac{A_{1}}{\cosh _{a}^{2}(\eta)}, \quad u(x, t)=\frac{A_{2}}{\cosh _{a}^{2}(\eta)},
$$

where $\eta=-(1 / 2 k \ln a)\left(x-\left(A_{1} / 3 p q\right) t\right)$.

Case 2. One has

$$
A_{2}=\frac{A_{1}}{2}(1 \pm i \sqrt{3}), \quad B=\frac{1}{2 k \ln a}, \quad v=\frac{A_{1}}{3 p q},
$$

where $A_{1}, k, p$, and $q$ are arbitrary constants. Substituting (34) into (22), we obtain new exact solution to (21),

$$
r(x, t)=\frac{A_{1}}{\cosh _{a}^{2}(\eta)}, \quad u(x, t)=\frac{A_{2}}{\cosh _{a}^{2}(\eta)},
$$

where $\eta=(1 / 2 k \ln a)\left(x-\left(A_{1} / 3 p q\right) t\right)$.

Remark 8. If we search the corresponding values for some parameters and make some transformations, in particular the solution (35) can be reduced to the solution (32) mentioned in [24].

\section{Conclusions and Remarks}

We consider generalized hyperbolic functions and new kinds of generalized hyperbolic function transformation to construct new exact solutions of nonlinear partial differential equations. This paper obtains 1-soliton solution to the generalized $\mathrm{KdV}$ equation and the coupled equal width wave equations. Our methods also can be applied to construct new exact solutions of other nonlinear partial differential equations.

\section{References}

[1] R. Hirota, "Exact N-soliton solutions of the wave equation of long waves in shallow-water and in nonlinear lattices," Journal of Mathematical Physics, vol. 14, no. 7, pp. 810-814, 1973.

[2] Y. Gurefe, Y. Pandir, and E. Misirli, "New exact solutions of stochastic KdV equation," Applied Mathematical Sciences, vol. 6, no. 65-68, pp. 3225-3234, 2012. 
[3] X. Wen, "Extended Jacobi elliptic function expansion solutions of variant Boussinesq equations," Applied Mathematics and Computation, vol. 217, no. 6, pp. 2808-2820, 2010.

[4] Y. Gurefe and E. Misirli, "Exp-function method for solving nonlinear evolution equations with higher order nonlinearity," Computers and Mathematics with Applications, vol. 61, no. 8, pp. 2025-2030, 2011.

[5] G. Ebadi and A. Biswas, "The $\left(G^{\prime} / G\right)$ method and 1-soliton solution of the Davey-Stewartson equation," Mathematical and Computer Modelling, vol. 53, no. 5-6, pp. 694-698, 2011.

[6] Y. Gurefe and E. Misirli, "New variable separation solutions of two-dimensional Burgers system," Applied Mathematics and Computation, vol. 217, no. 22, pp. 9189-9197, 2011.

[7] N. A. Kudryashov, "Simplest equation method to look for exact solutions of nonlinear differential equations," Chaos, Solitons and Fractals, vol. 24, no. 5, pp. 1217-1231, 2005.

[8] N. K. Vitanov, "Modified method of simplest equation: Powerful tool for obtaining exact and approximate traveling-wave solutions of nonlinear PDEs," Communications in Nonlinear Science and Numerical Simulation, vol. 16, no. 3, pp. 1176-1185, 2011.

[9] C. S. Liu, "Applications of complete discrimination system for polynomial for classifications of traveling wave solutions to nonlinear differential equations," Computer Physics Communications, vol. 181, no. 2, pp. 317-324, 2010.

[10] Y. Gurefe, A. Sonmezoglu, and E. Misirli, "Application of an irrational trial equation method to high-dimensional nonlinear evolution equations," Journal of Advanced Mathematical Studies, vol. 5, no. 1, pp. 41-47, 2012.

[11] Y. Pandir, Y. Gurefe, U. Kadak, and E. Misirli, "Classifications of exact solutions for some nonlinear partial differential equations with generalized evolution," Abstract and Applied Analysis, vol. 2012, Article ID 478531, 16 pages, 2012.

[12] Y. Xie and J. Tang, "The superposition method in seeking the solitary wave solutions to the KdV-Burgers equation," Pramana, vol. 66, no. 3, pp. 479-483, 2006.

[13] N. A. Kudryashov, "One method for finding exact solutions of nonlinear differential equations," Communications in Nonlinear Science and Numerical Simulation, vol. 17, no. 6, pp. 2248-2253, 2012.

[14] Y. Pandir, Y. Gurefe, and E. Misirli, "A new approach to Kudryashovs method for solving some nonlinear physical models," International Journal of Physical Sciences, vol. 7, pp. 2860-2866, 2012.

[15] N. A. Kudryashov and S. G. Prilipko, "Exact solutions of the generalized $\mathrm{K}(\mathrm{m}, \mathrm{m})$ equations," Communications in Nonlinear Science and Numerical Simulation, vol. 16, no. 3, pp. 1107-1113, 2011.

[16] G. Ebadi and A. Biswas, "Application of the $\left(G^{\prime} / G\right)$-expansion method for nonlinear diffusion equations with nonlinear source," Journal of the Franklin Institute, vol. 347, no. 7, pp. 1391-1398, 2010.

[17] G. Ebadi and A. Biswas, "The $\left(G^{\prime} / G\right)$ method and topological soliton solution of the $\mathrm{K}(\mathrm{m}, \mathrm{n})$ equation," Communications in Nonlinear Science and Numerical Simulation, vol. 16, no. 6, pp. 2377-2382, 2011.

[18] G. Ebadi, A. H. Kara, M. D. Petkovic, A. Yildirim, and A. Biswas, "1-Soliton solution of the generalized KdV equation with generalized evolution," Proceedings of the Romanian Academy A, vol. 13, pp. 215-224, 2012.
[19] A. Biswas, M. D. Petković, and D. Milović, "Topological exact soliton solution of the power law KdV equation," Applied Mathematics and Computation, vol. 217, no. 4, pp. 1780-1784, 2010.

[20] Y. J. Ren and H. Q. Zhang, "New generalized hyperbolic functions and auto-Bäcklund transformation to find new exact solutions of the (2+1)-dimensional NNV equation," Physics Letters A, vol. 357, no. 6, pp. 438-448, 2006.

[21] E. Yomba, "Generalized hyperbolic functions to find solitonlike solutions for a system of coupled nonlinear Schrödinger equations," Physics Letters A, vol. 372, no. 10, pp. 1612-1618, 2008.

[22] Z. I. A. Al-Muhiameed and E. A.-B. Abdel-Salam, "Generalized hyperbolic function solution to a class of nonlinear Schrödinger-type equations," Journal of Applied Mathematics, vol. 2012, Article ID 265348, 15 pages, 2012.

[23] M. S. Ismail and A. Biswas, "1-Soliton solution of the generalized KdV equation with generalized evolution," Applied Mathematics and Computation, vol. 216, no. 5, pp. 1673-1679, 2010.

[24] A. H. A. Ali, A. A. Soliman, and K. R. Raslan, "Soliton solution for nonlinear partial differential equations by cosine-function method," Physics Letters A, vol. 368, no. 3-4, pp. 299-304, 2007. 


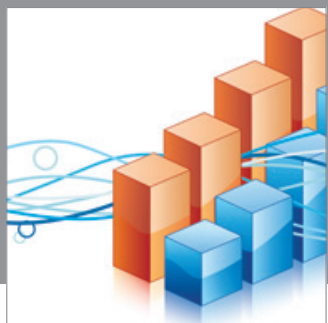

Advances in

Operations Research

mansans

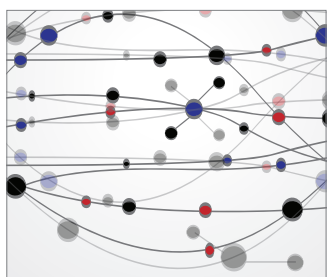

The Scientific World Journal
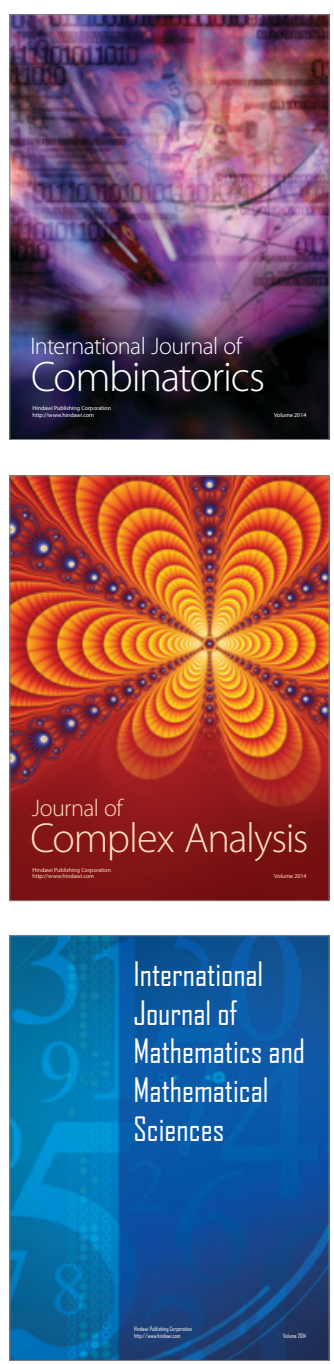
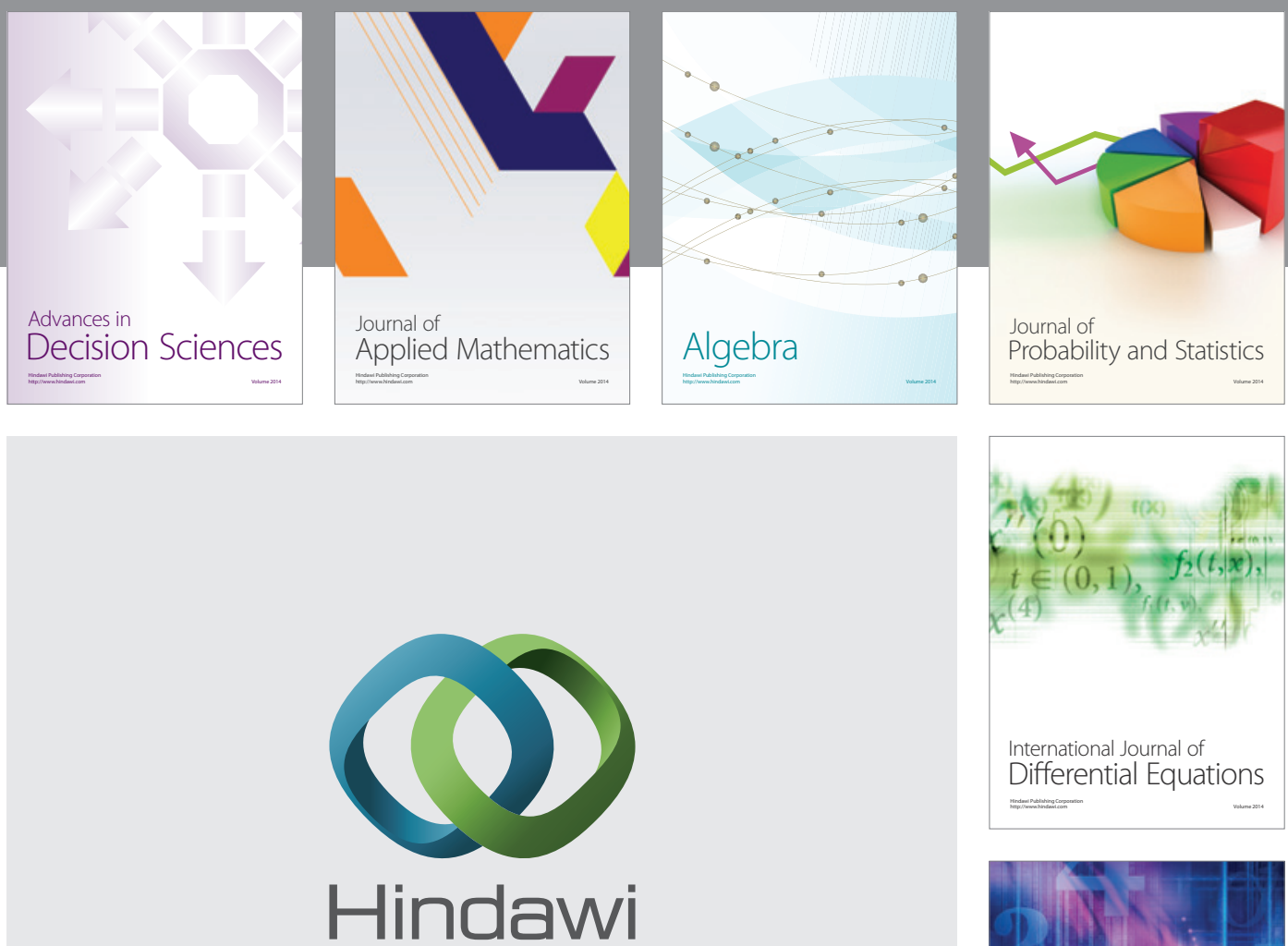

Submit your manuscripts at http://www.hindawi.com
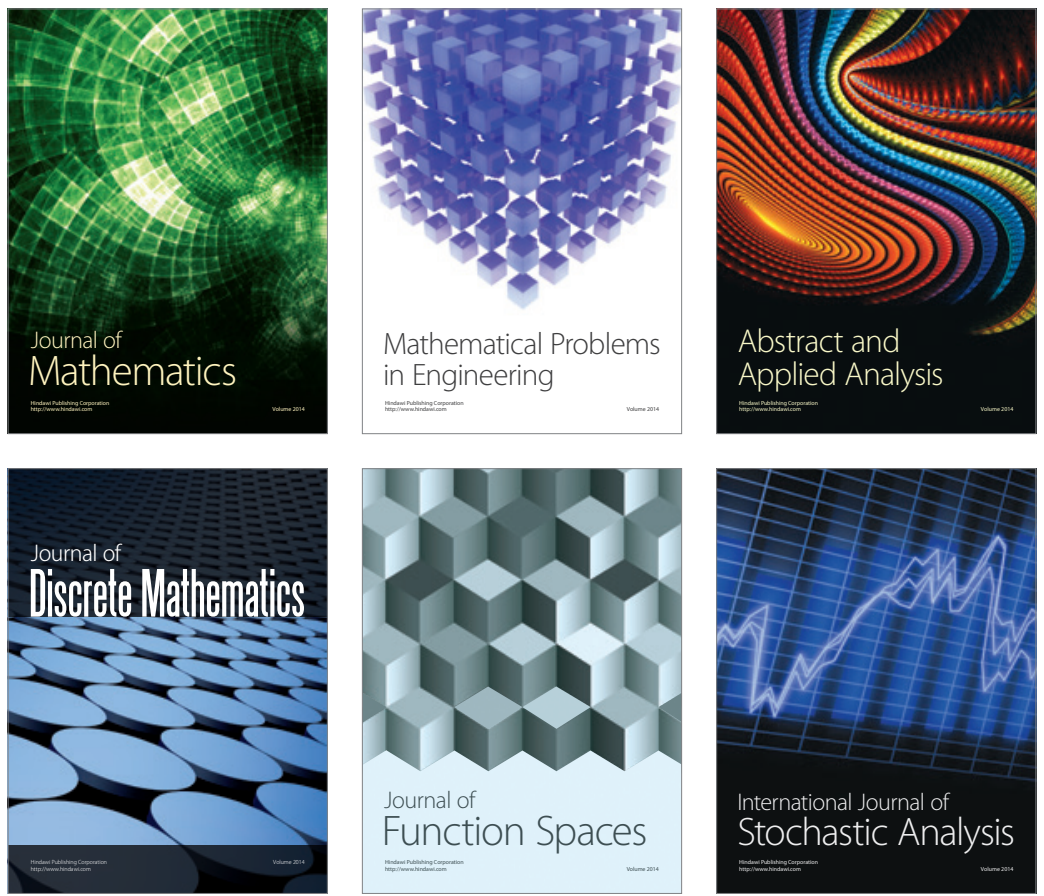

Journal of

Function Spaces

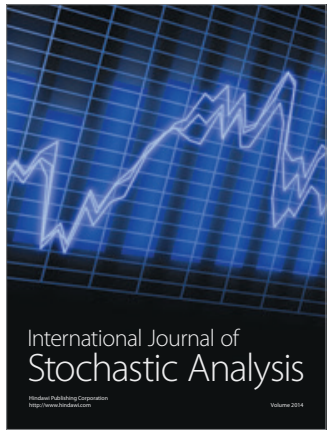

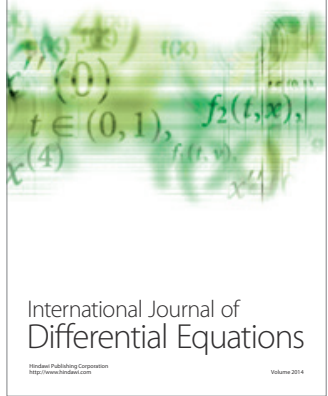
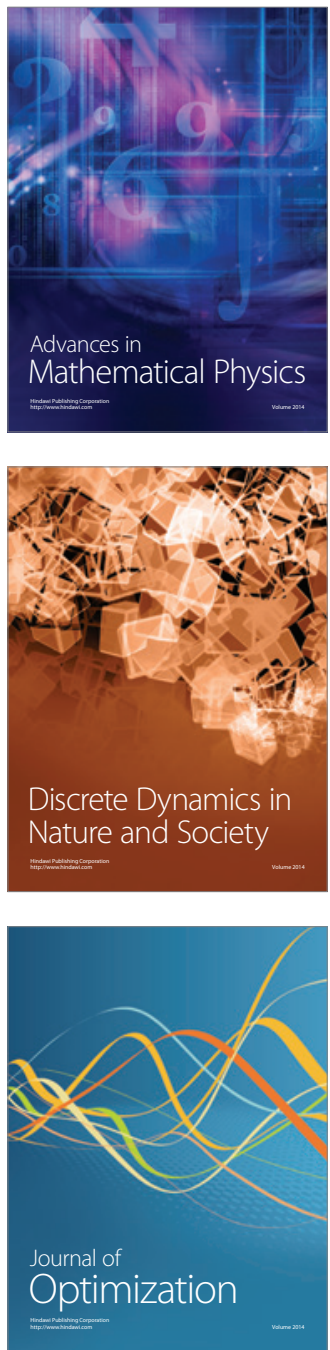